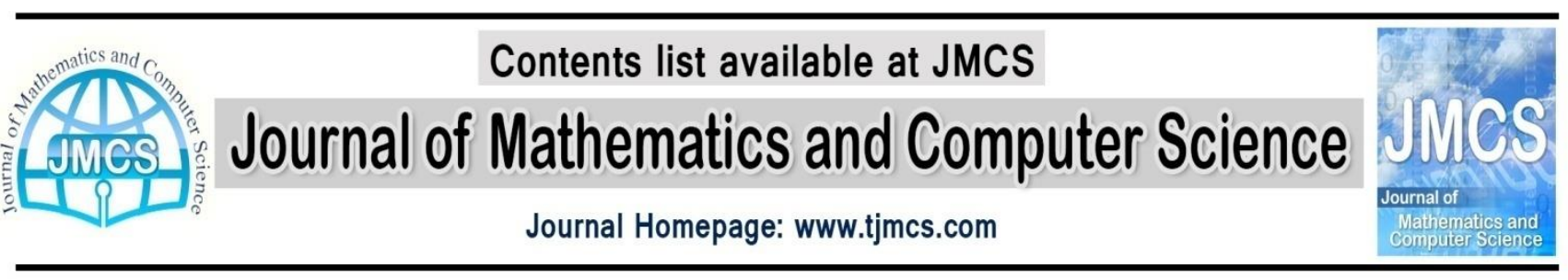

\title{
An Improved Model of Brain Emotional Learning Algorithm Based on Interval Knowledge
}

\author{
Yousef Sharafi ${ }^{1, *}$, Saeed Setayeshi ${ }^{2}$, Alireza Falahiazar ${ }^{1,+}$ \\ ${ }^{1}$ Computer Department of Islamic Azad University Science and Research Branch, Tehran, Iran \\ y.sharafi@srbiau.ac.ir \\ +a_falahiazar@srbiau.ac.ir \\ ${ }^{2}$ Faculty of Nuclear Engineering and Physics, Amirkabir University, Tehran, Iran \\ setayesh@aut.ac.ir
}

Article history:

Received July 2014

Accepted October 2014

Available online November 2014

\begin{abstract}
The brain emotional learning algorithm inspired by a reduced system of a computational model simulates the brain learning performance quite simply with mimicking mammalian brains. The present paper endeavors to come forward to an improved model of the emotional learning algorithm based on the interval knowledge. In this proposed model, based on the interval knowledge, the weights of the amygdala and orbitofrontal sections will be updated. Eventually, the results of implementing and performing the improved brain emotional learning algorithm will be presented to be compared with the original version of the algorithm to Prediction the chaotic time series, Lorenz and Rossler, about which a noticeable improvement in its precision, accuracy and speed of convergence of the final results is reported.
\end{abstract}

Keywords: Brain Emotional Learning Algorithm, Interval Knowledge, Chaotic Time Series, Prediction

\section{Introduction}

Emotional computation is considered as an interdisciplinary field, including computer science, psychology and cognitive science. In the decision-making process not only computations and logic which are performed by the upper tissue of the brain but the sensations whose origins are in the cerebellum, and midbrain is involved. The brain learning and its applications can be considered as an almost new field of research. The undertaken researches about the aforementioned area reveal that the advocates' claims and contentions about brain learning, without a doubt, were of great value and were worth devoting some time and energy. With regard to above mentioned issue, various methods of education based on the brain learning have been proposed. The brain can act in different ways simultaneously. Additionally, the brain constantly processes and monitors the thoughts, excitements, 
and images. The brain is a physiological-neural system, which interacting and exchanging information with the environment. Human being is not aware of some brain applications, take control of breathing and some other inevitable activities as examples. Some of the applications of neural networks are for use in stock price prediction [1], bankruptcy Prediction [2], Detect dos-attacks [3] and controlling the False Alarm in an Intrusion Tolerant Database System [4]. Since the brain has various performances, learning process can be complex and different as well. Neural networks trained with gradient descent based algorithms [5] and evolutionary algorithms [6-7]. In this regard, not only the considerable importance of learning fields and material, but also learning methodologies are being stressed. Reptiles react to symbols of chemical, touch, and sight senses and their reactions have already been determined in accordance with their bodies. There are some excitements originated from determined activities in specific areas of the brain called Limbic System. Some significant areas are located in the cortex of the brain, namely Amygdala, Orbitofrontal, hypothalamus, hippocampus, thalamus, etc. Not only are not all excitements merely related to Limbic, but also it has been shown that some Limbic systems are not related to the excitements directly [8-11]. In this paper, applying the interval knowledge, it is being endeavored to introduce a version of the brain emotional learning algorithm which shows better results in prediction the chaotic time series in comparison with the original version of the brain emotional learning algorithm. This paper is organized as follows: Firstly, the interval knowledge will be reviewed, and then the brain emotional learning algorithm will be investigated and the proposed method will be suggested, and eventually the results of the proposed method will be compared with the original version of the brain emotional learning algorithm so that Lorenz and Rossler chaotic time series will be forecasted.

\section{Interval Knowledge}

In this section, a type of application of interval knowledge in neural network is being presented whose concepts are going to be used to create the improved proposed algorithm. Rough Neural Network is being utilized to estimate the functions based on Lingras model. With any input data and real output values, Rough Neural Network can be implemented [13], figures 1 and 2 shows the structure of the Rough Neuron.

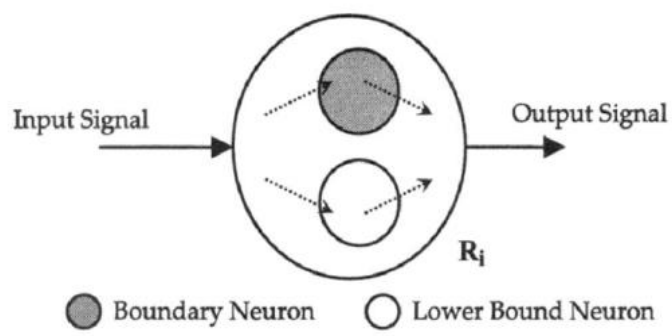

Figure 1. Rough Neuron [14]

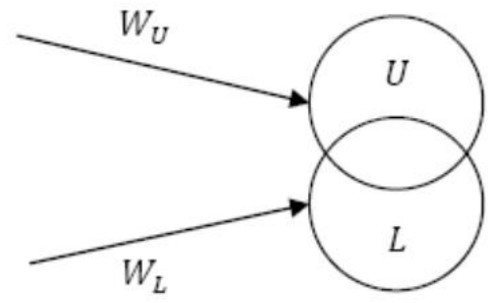

Figure 2. The Structure of the rough neuron with upper weight and lower weight 
Where $U$ is the upper boundary neuron and $L$ represents the lower boundary neuron. One sample of Rough Neural Network is being shown in figure 3. Rough hidden layer neurons consist of upper and lower boundary neurons. Having calculated the average, the output of each rough neuron in this layer is obtained. The single neuron of the output layer in this model is a conventional one. In this model, having announced the error based on first rank gradient descent, learning takes place [13].
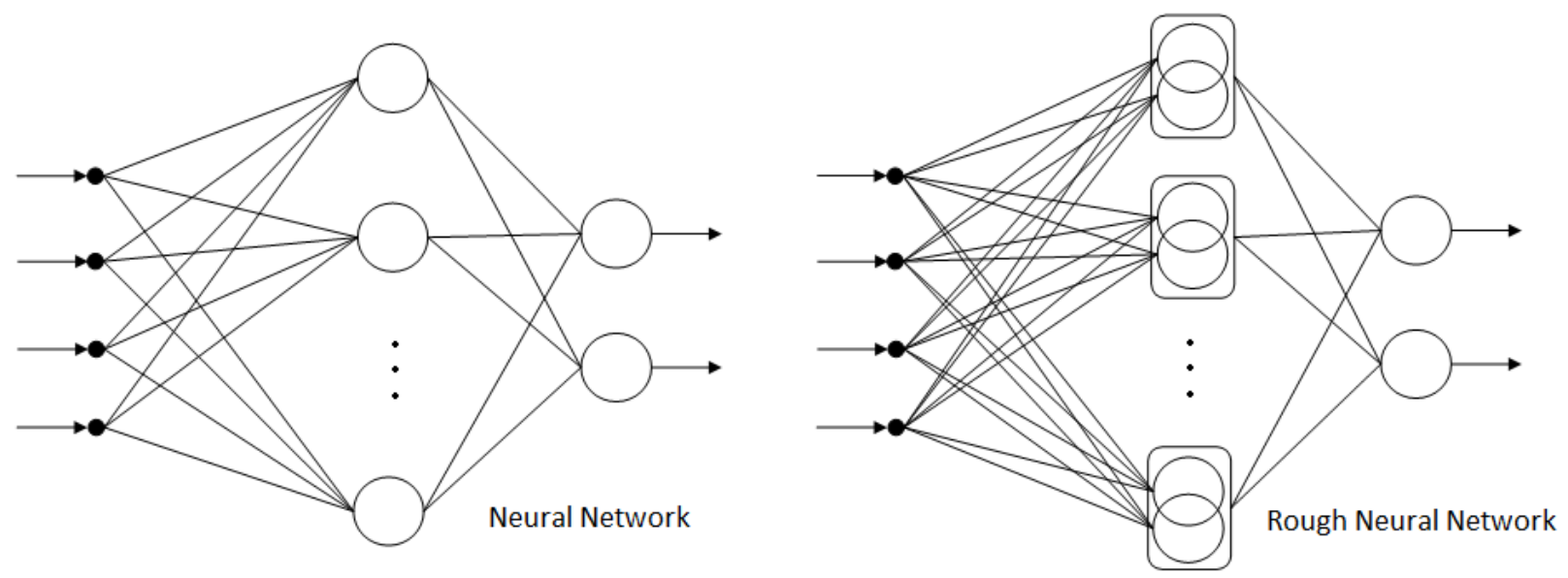

Figure 3. Comparison between the conventional neural networks and rough neural networks

\section{Brain Emotional Learning Algorithm}

Limbic system is shown in figure 4 and computational model of the brain emotional learning algorithm is shown in figure 5 .

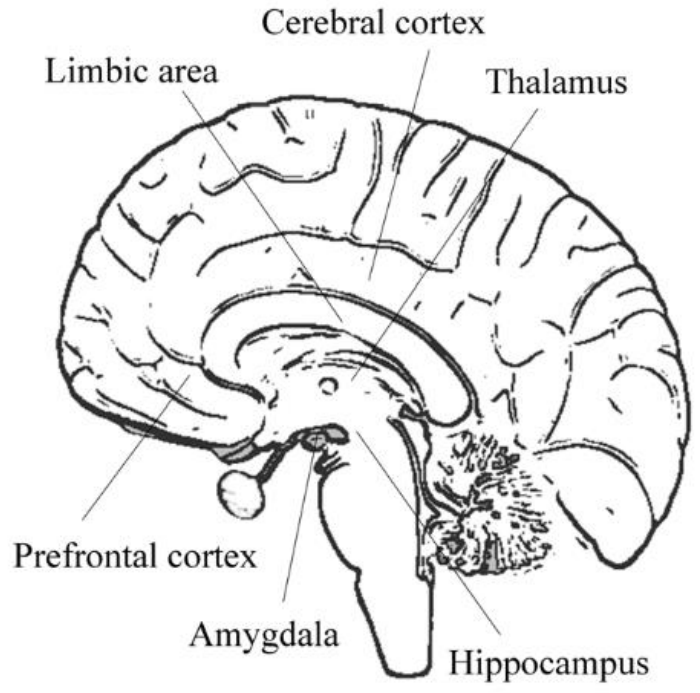

Figure 4. The limbic system in the brain [17]

The given system is categorized into two sections, namely orbitofrontal cortex and amygdala. Confirmatory reward signal is applied to the system, and for each $S$ stimulation like thalamic stimulation tie, an $A$ tie exists. In addition, an $O$ tie exists for everyone except for the thalamic stimulation tie. $E$ tie 
is the output tie of the model which in actual fact collects the outputs of all $A$ ties and then subtract the total amount of deterrent ties. The amygdala section plays a crucial role in controlling the emotional activities such as friendship, love and kindness, temper, fear, invasion and anger. The amygdala is the center of noticing the dangers and is of a great importance for human's survival. The amygdala section is trained to predict the next reactions and react to the reward. Orbitofrontal interferes when the prediction made by the amygdala and the reward are not consonant with each other. Consequently, based on the former learning, it endeavors to remove this dissonance. The most significant part of learning algorithm is defining the reward function. Reinforced reward signal is a function of other signals considered as an evaluation function ([12], [15], and [16]).

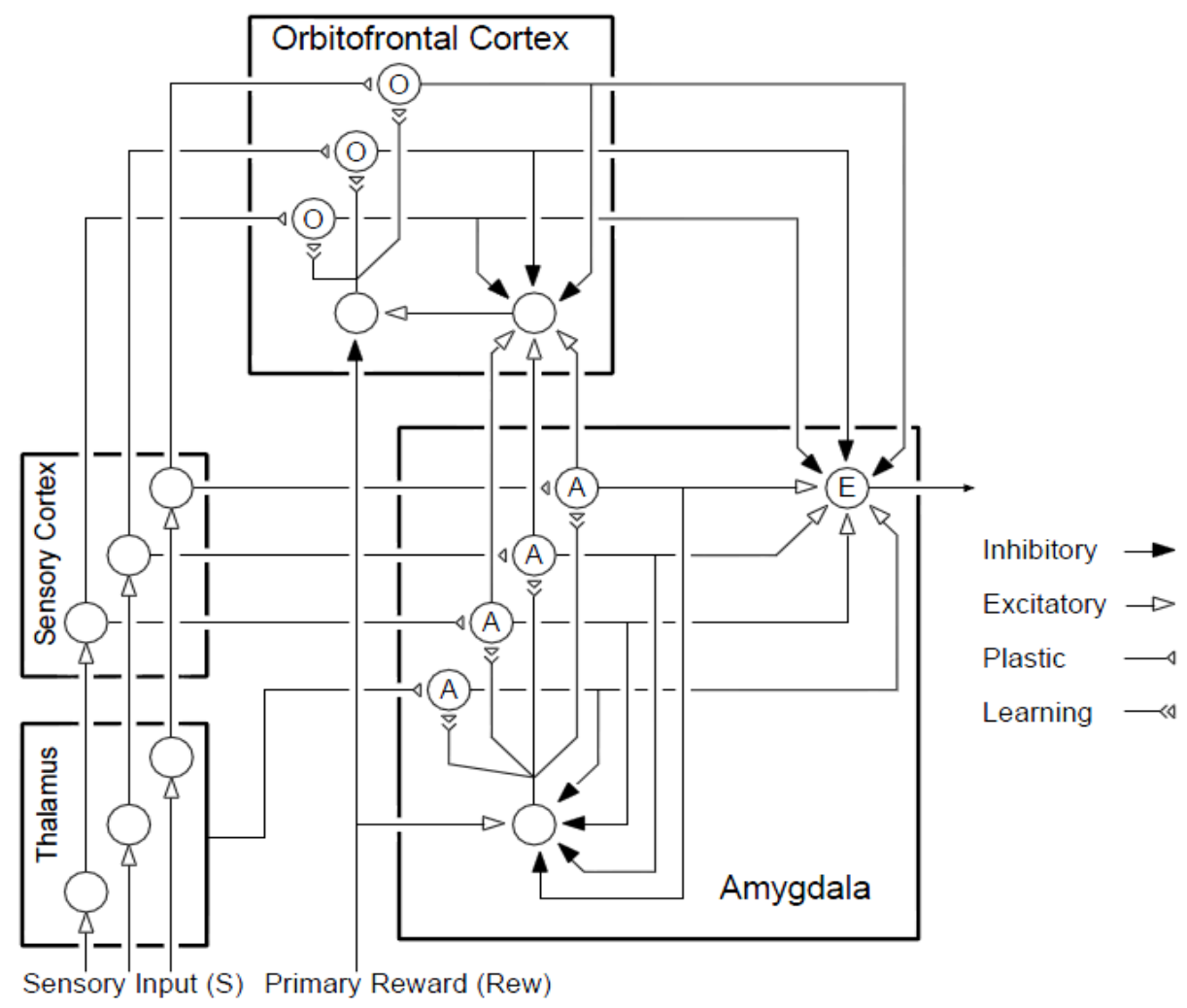

Figure 5. Computational model for brain emotional learning [12]

The output of ties in the amygdala and the orbitofrontal tissue, and the final output of the computational model of the brain emotional learning algorithm are calculated by equations 1-3 respectively.

$$
\begin{gathered}
O_{i}=S_{i} * W_{i} \\
A_{i}=S_{i} * V_{i} \\
E=\sum_{i} A_{i}-\sum_{i} O_{i}
\end{gathered}
$$

The amygdala is responsible for stimulation and orbitofrontal has a deterrent role. In this model, these $V_{i}$ are the weights of amygdala and $W_{i}$ are the weights of Orbitofrontal; $A_{i}$ and $O_{i}$ are the output of the ties in the amygdala and orbitofrontal tissue respectively; $S_{i}$ are the sensory input, E is the final output 
of the computational model. Equations 4 and 5 are being used to reconcile the weights in learning process [12].

$$
\begin{gathered}
\Delta V_{i}=\alpha\left(\mathrm{S}_{i} \max \left(0, \mathrm{REW}-\sum_{j} A_{j}\right)\right. \\
\Delta W_{i}=\beta\left(\mathrm{S}_{i} \sum_{j}\left(\mathrm{O}_{j}-\mathrm{REW}\right)\right)
\end{gathered}
$$

According to the abovementioned equations, $\alpha$ and $\beta$ are the coefficients of the weights of the amygdala and orbitofrontal and $R E W$ is the reward. $A_{t h}$ Which can be calculated by equation 6 is the value which moves from thalamus towards amygdala [12].

$$
A_{t h}=\max \left(S_{1}, S_{2}, \ldots, S_{n}\right)
$$

$n$ is the number of inputs of thalamus section; $S_{i}$ is the i-th sensory input. In figure 6 , a very simple structure of connections among the major sections of learning computational system is easily seen. In proposed method, applying the interval knowledge, the weights of the amygdala and orbitofrontal are being taught.

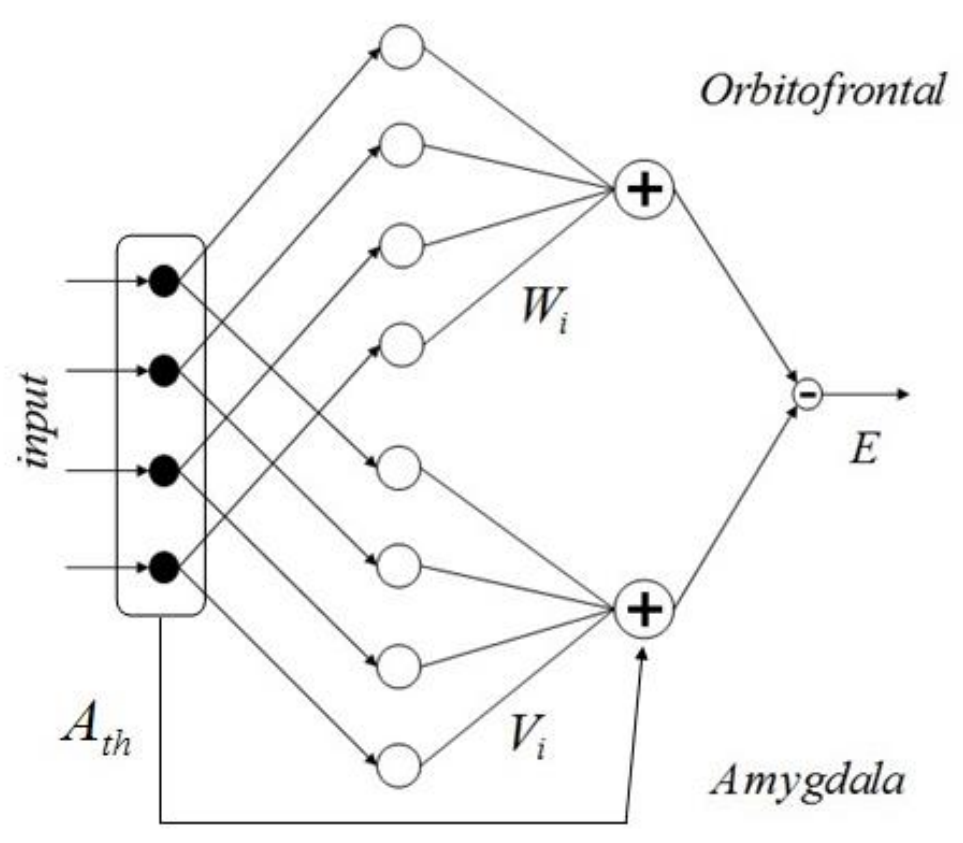

Figure 6. Simple structure of the brain emotional learning 


\section{The Propose Method}

As can be seen in figure 7, the weights of amygdala and orbitofrontal are changed into intervals. $V_{i}$ is the weight of the amygdala and $W_{i}$ is the weight of orbitofrontal which have lower and upper boundaries. According to figure 8,

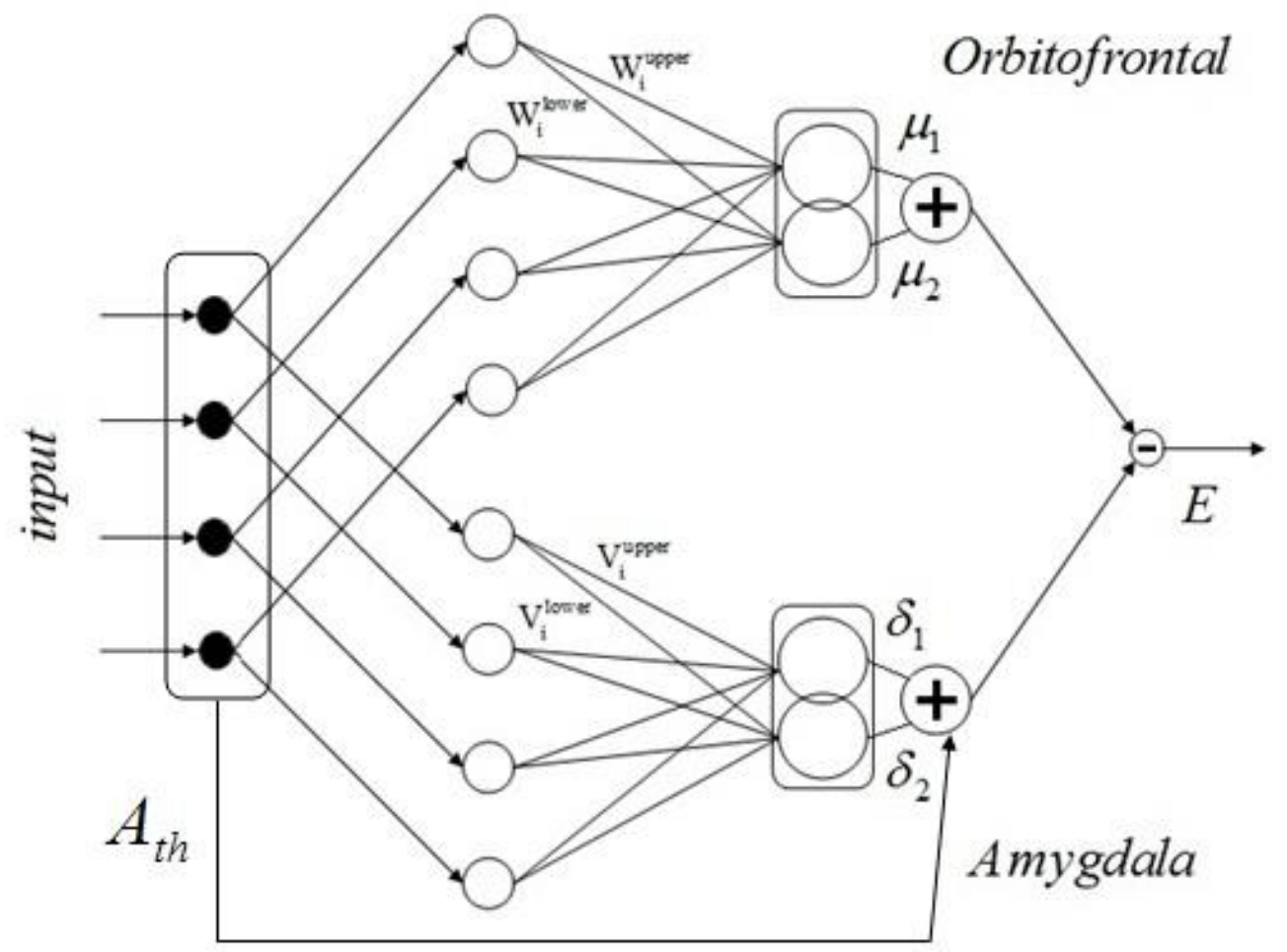

Figure 7. The Structure of the proposed method for brain emotional Learning

Therefore, the outputs of the amygdala and orbitofrontal tissue will include lower and upper boundaries.
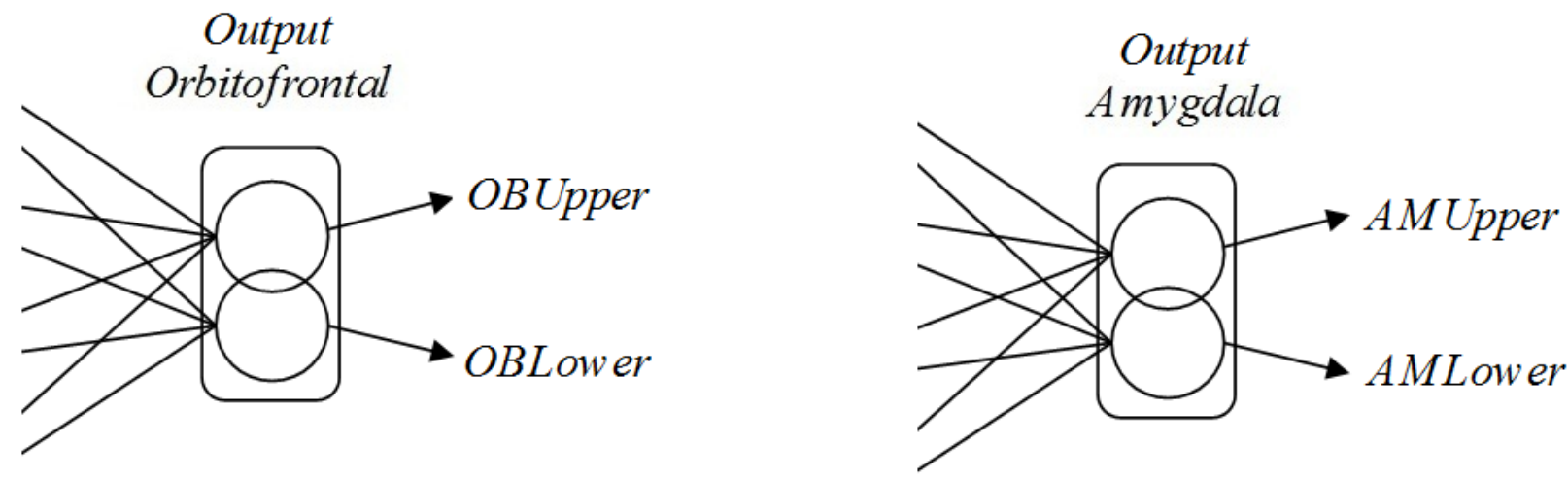

Figure 8. The output neurons of the amygdala and orbitofrontal 
On the other hand, the lower and upper outputs of the amygdala will multiply by $\delta_{1}$ and $\delta_{2}$ respectively, and eventually with accordance with equation 10, their total will be considered as the output of the amygdala. Similarly, the lower and upper outputs of orbitofrontal will multiply by $\mu_{1}$ and $\mu_{2}$ respectively and eventually with accordance with equation 9 , their total will be considered as the output of orbitofrontal.

$$
\begin{gathered}
\text { OBUpper }=\sum_{i} S_{i} * W_{i}^{\text {Upper }} \\
\text { OBLower }=\sum_{i} S_{i} * W_{i}^{\text {Lower }} \\
\text { OutputOB }=\text { OBUpper } * \delta_{1}+\text { OBLower } * \delta_{2} \\
\text { OutputAM }=\text { AMUpper } * \mu_{1}+\text { AMLower } * \mu_{2}
\end{gathered}
$$

To update $\delta_{1}$ and $\delta_{2}$ parameters in the amygdala, $\mu_{1}$ and $\mu_{2}$ in orbitofrontal section, equations 11-16 must be followed. $\eta_{1}, \eta_{2}, \eta_{3}$ and $\eta_{4}$ are the rates of the output parameters of the amygdala and orbitofrontal.

$$
\begin{gathered}
e=\text { Target }-\mathrm{E} \\
p=\frac{1}{2}(\mathrm{e})^{2} \\
\delta_{1}(t)=\delta_{1}(t-1)+\eta_{1} * \frac{\partial p}{\partial \delta_{1}}=\delta_{1}(t-1)+\eta_{1} * \frac{\partial p}{\partial e} * \frac{\partial e}{\partial E} * \frac{\partial E}{\partial A M} * \frac{\partial A M}{\partial \delta_{1}} \\
\delta_{1}(t-1)+\eta_{1} *(e) *(-1) *(1) *(A M U p p e r) \\
\delta_{2}(t)=\delta_{2}(t-1)+\eta_{2} * \frac{\partial p}{\partial \delta_{2}}=\delta_{2}(t-1)+\eta_{2} * \frac{\partial p}{\partial e} * \frac{\partial e}{\partial E} * \frac{\partial E}{\partial A M} * \frac{\partial A M}{\partial \delta_{2}} \\
\delta_{2}(t-1)+\eta_{2} *(e) *(-1) *(1) *(A M L o w e r) \\
\mu_{1}(t)=\mu_{1}(t-1)+\eta_{3} * \frac{\partial p}{\partial \mu_{1}}=\mu_{1}(t-1)+\eta_{3} * \frac{\partial p}{\partial e} * \frac{\partial e}{\partial E} * \frac{\partial E}{\partial O B} * \frac{\partial O B}{\partial \mu_{1}} \\
\mu_{1}(t-1)+\eta_{3} *(e) *(-1) *(1) *(O B U p p e r) \\
\mu_{2}(t)=\mu_{2}(t-1)+\eta_{4} * \frac{\partial p}{\partial \mu_{2}}=\mu_{2}(t-1)+\eta_{4} * \frac{\partial p}{\partial e} * \frac{\partial e}{\partial E} * \frac{\partial E}{\partial O B} * \frac{\partial O B}{\partial \mu_{2}} \\
\mu_{2}(t-1)+\eta_{4} *(e) *(-1) *(1) *(O B L o w e r)
\end{gathered}
$$

To learn the weights of lower and upper bounds of amygdala and orbitofrontal, equations 4 and 5 must be followed. The only difference is that the $V_{i}$ weight can be taught by learning the weights of $V_{i}^{\text {Upper }}$ and $V_{i}^{\text {Lower }}$ separately, and $W_{i}$ weight is also the same. 


\section{Experimental Results}

In this part of the paper, the results of implementing and performing the improved algorithm to the Prediction of the Lorenz attractor and Rossler attractor chaotic time series are investigated, and eventually there will be a comparison between the improved algorithm and the original version of this algorithm. Rossler attractor chaotic time series are presented by the following equations,

$$
\begin{gathered}
\frac{d x(t)}{d t}=-\sigma_{L} x(t)+\sigma_{L} y(t) \\
\frac{d y(t)}{d t}=\gamma_{L} x(t)-y(t)+\mathrm{x}(\mathrm{t}) \mathrm{z}(\mathrm{t}) \\
\frac{d z(t)}{d t}=-b_{L} z(t)+x(t) y(t)
\end{gathered}
$$

where $\sigma_{L}, \gamma_{L}, b_{L}$ and do not have dimensions and present the dynamic Lorenz attractor. If the value of $\gamma_{L}$ is 24.74 more than a critical value, $\sigma_{L}=10$ and $b_{L}=3 / 8$ will have the chaotic behavior system. Almost 8000 samples of Lorenz attractor time series are shown in figure 9.

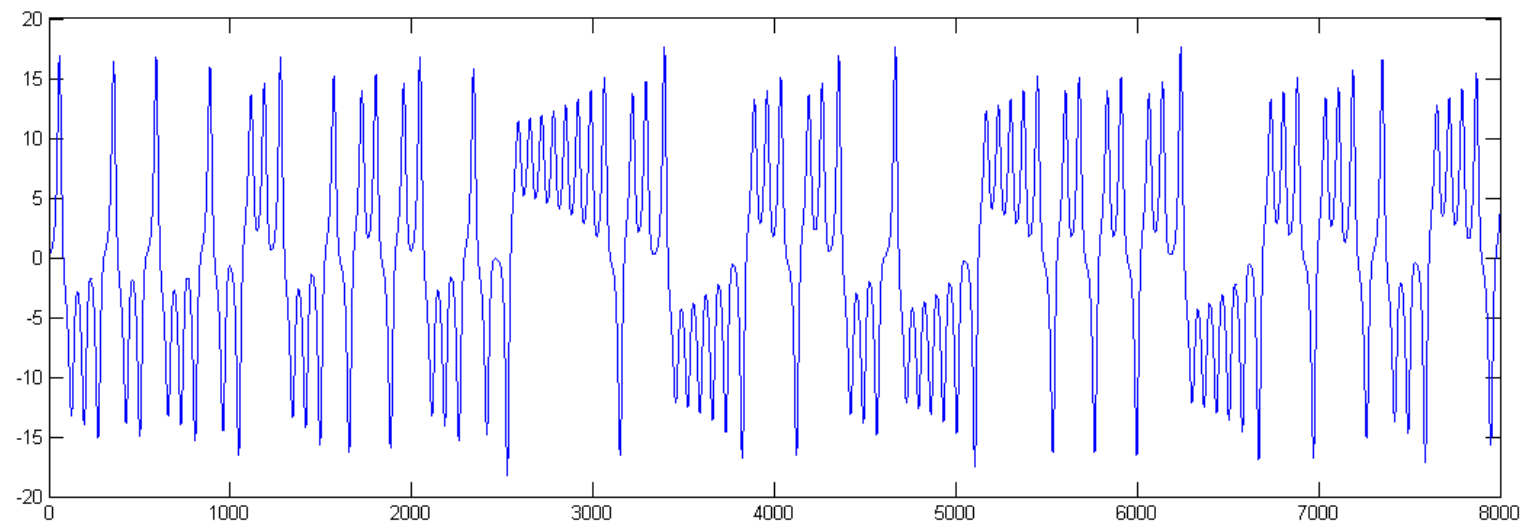

Figure 9. Lorenz chaotic time series

Another time series which has been studied is the Rossler attractor presented by the following equations,

$$
\begin{gathered}
\frac{d x(t)}{d t}=-z(t)-y(t) \\
\frac{d y(t)}{d t}=x(t)+a * y(t) \\
\frac{d z(t)}{d t}=b+z(t) *(x(t)-c)
\end{gathered}
$$

Where $a=0.15, b=0.20, c=10$ and do not have dimensions. 8000 samples of Rossler attractor time series are shown in figure 10 . 


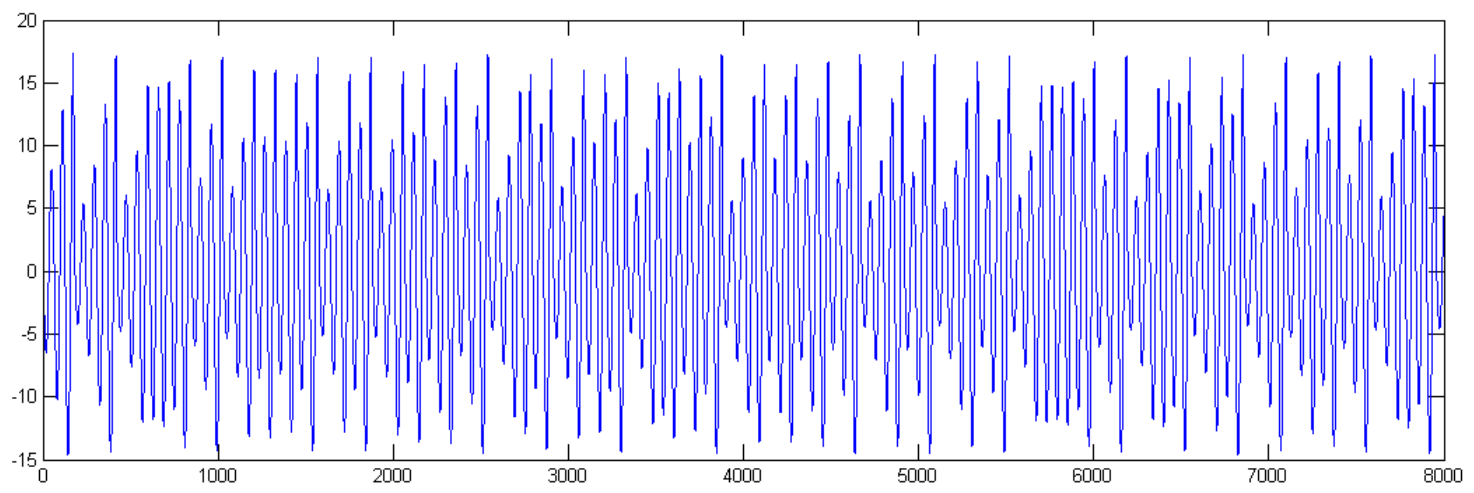

Figure 10. Rossler chaotic time series

The results of comparing the proposed algorithm and the original version [12] to prediction the Lorenz and Rossler time series are reported in tables 1-2 and figures 11-14. One of the noticeable points in the reported results of this paper is the point that with the fewest testing samples, prediction is done carefully.

Table 1. The results of comparison of the proposed method and moren[12] for prediction of lorenz chaotic time series

\begin{tabular}{|c|c|c|c|c|c|c|}
\hline Algorithm & Max Epoch & Train & Test & Number of Sample & MSE - Train & MSE - Test \\
\hline Moren[12] & 150 & $5 \%$ & $95 \%$ & 8000 & 0.000561865000 & 0.000541915300 \\
\hline Proposed & 150 & $5 \%$ & $95 \%$ & 8000 & $\mathbf{0 . 0 0 0 2 3 6 7 9 8 3 0 0}$ & $\mathbf{0 . 0 0 0 2 2 9 8 8 8 3 0 0}$ \\
\hline Moren[12] & 150 & $10 \%$ & $90 \%$ & 8000 & 0.000277757000 & 0.000303022100 \\
\hline Proposed & 150 & $10 \%$ & $90 \%$ & 8000 & $\mathbf{0 . 0 0 0 1 3 4 2 1 8 8 0 0}$ & $\mathbf{0 . 0 0 0 1 4 0 8 0 4 8 0 0}$ \\
\hline Moren[12] & 150 & $15 \%$ & $85 \%$ & 8000 & 0.000138474100 & 0.000130332100 \\
\hline roposed & 150 & $15 \%$ & $85 \%$ & 8000 & $\mathbf{0 . 0 0 0 0 9 9 8 3 8 3 0 0}$ & $\mathbf{0 . 0 0 0 0 9 1 6 0 4 1 0 0}$ \\
\hline
\end{tabular}

Table 2. The results of comparison of the proposed method and moren[12] for prediction of rossler chaotic time series

\begin{tabular}{|c|c|c|c|c|c|c|}
\hline Algorithm & Max Epoch & Train & Test & Number of Sample & MSE - Train & MSE - Test \\
\hline Moren[12] & 150 & $5 \%$ & $95 \%$ & 8000 & 0.000215253600 & 0.000225777400 \\
\hline Proposed & 150 & $5 \%$ & $95 \%$ & 8000 & $\mathbf{0 . 0 0 0 1 5 4 3 3 3 1 0 0}$ & $\mathbf{0 . 0 0 0 1 6 0 3 3 6 4 0 0}$ \\
\hline Moren[12] & 150 & $10 \%$ & $90 \%$ & 8000 & 0.000109057700 & 0.000111138200 \\
\hline Proposed & 150 & $10 \%$ & $90 \%$ & 8000 & $\mathbf{0 . 0 0 0 0 8 4 3 1 4 0 0 0}$ & $\mathbf{0 . 0 0 0 0 8 6 3 4 3 2 0 0}$ \\
\hline Moren[12] & 150 & $15 \%$ & $85 \%$ & 8000 & 0.000062907700 & 0.000062565300 \\
\hline Proposed & 150 & $15 \%$ & $85 \%$ & 8000 & $\mathbf{0 . 0 0 0 0 6 0 3 5 4 2 0 0}$ & $\mathbf{0 . 0 0 0 0 6 1 5 4 7 2 1 0}$ \\
\hline
\end{tabular}




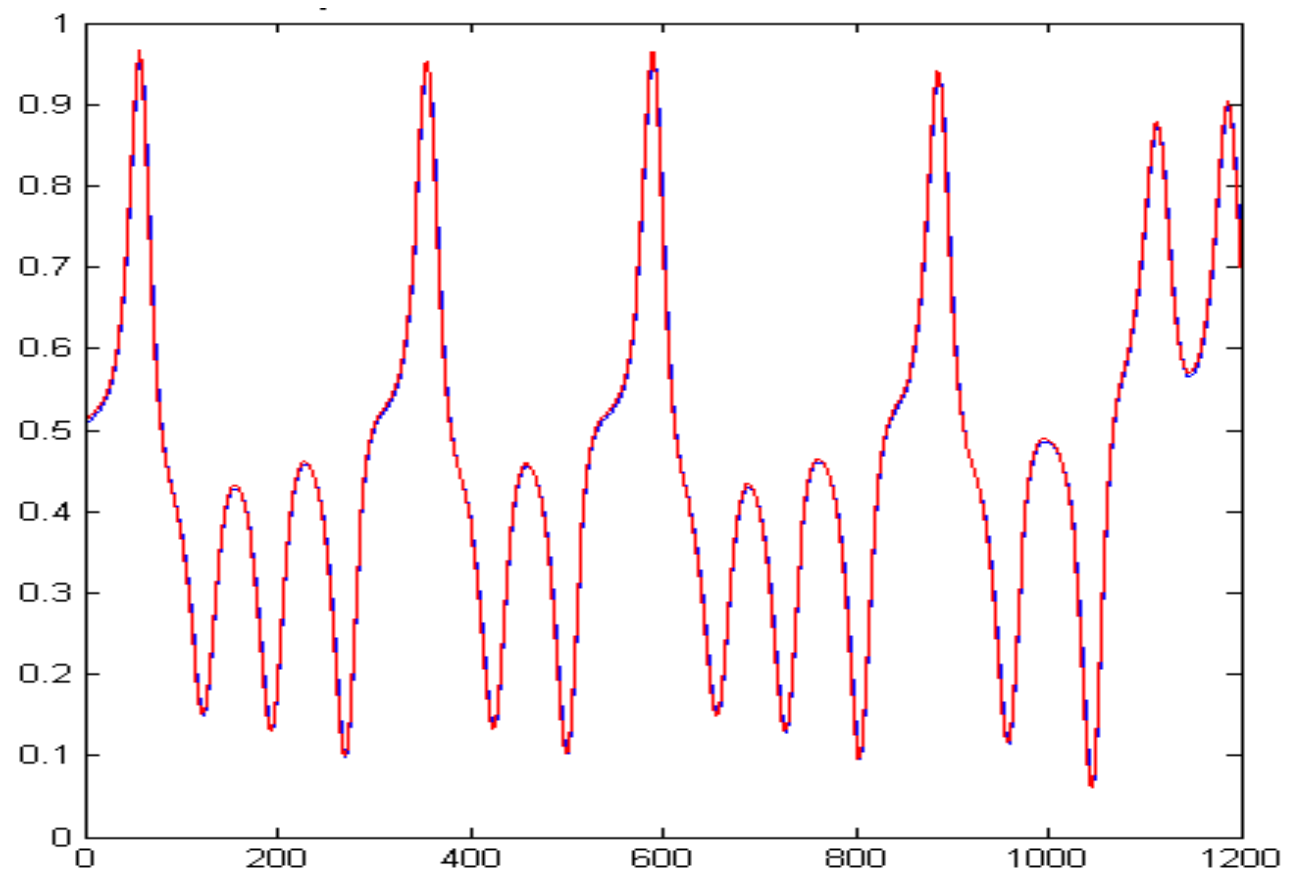

Figure 11. The predicted values and the target values of lorenz time series (Train)

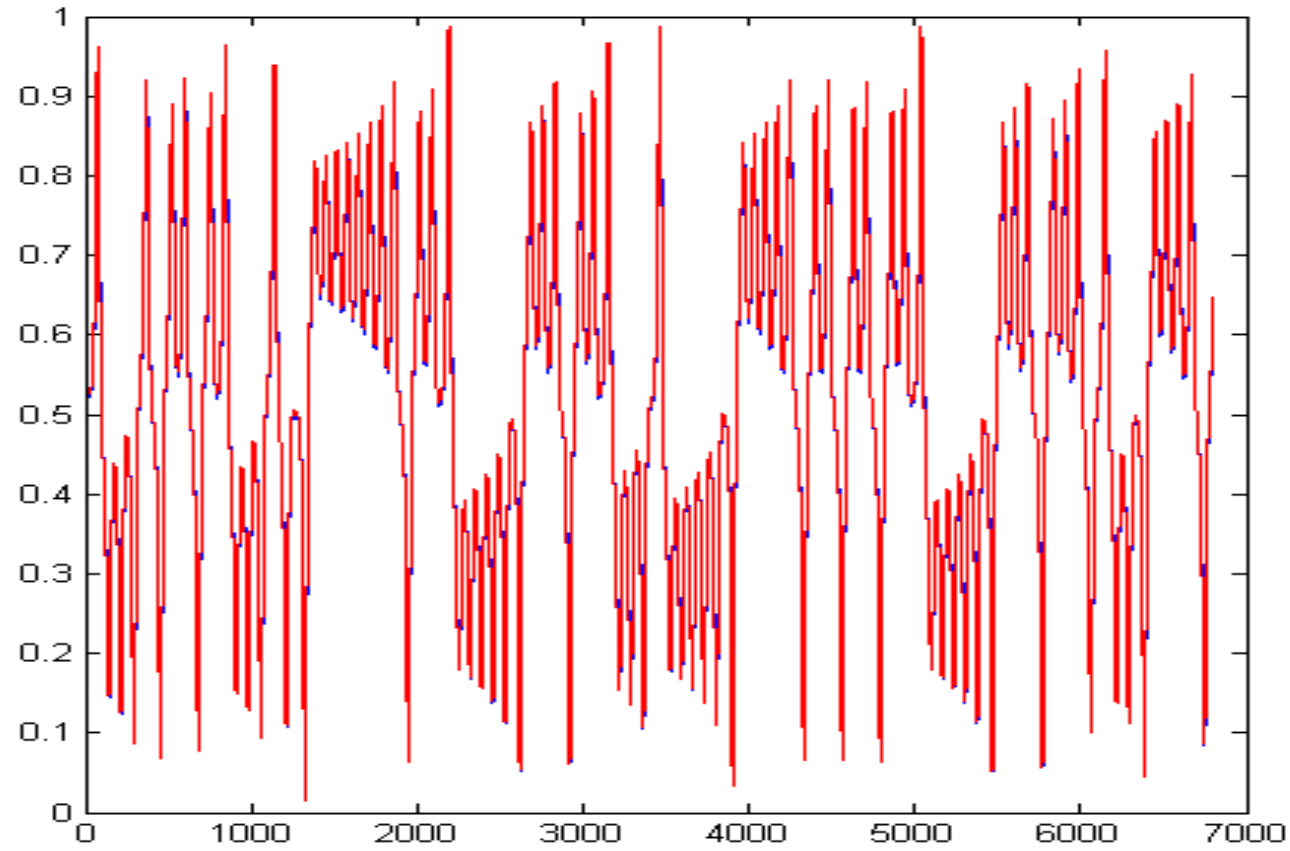

Figure 12. The predicted values and the target values of lorenz time series (Test) 


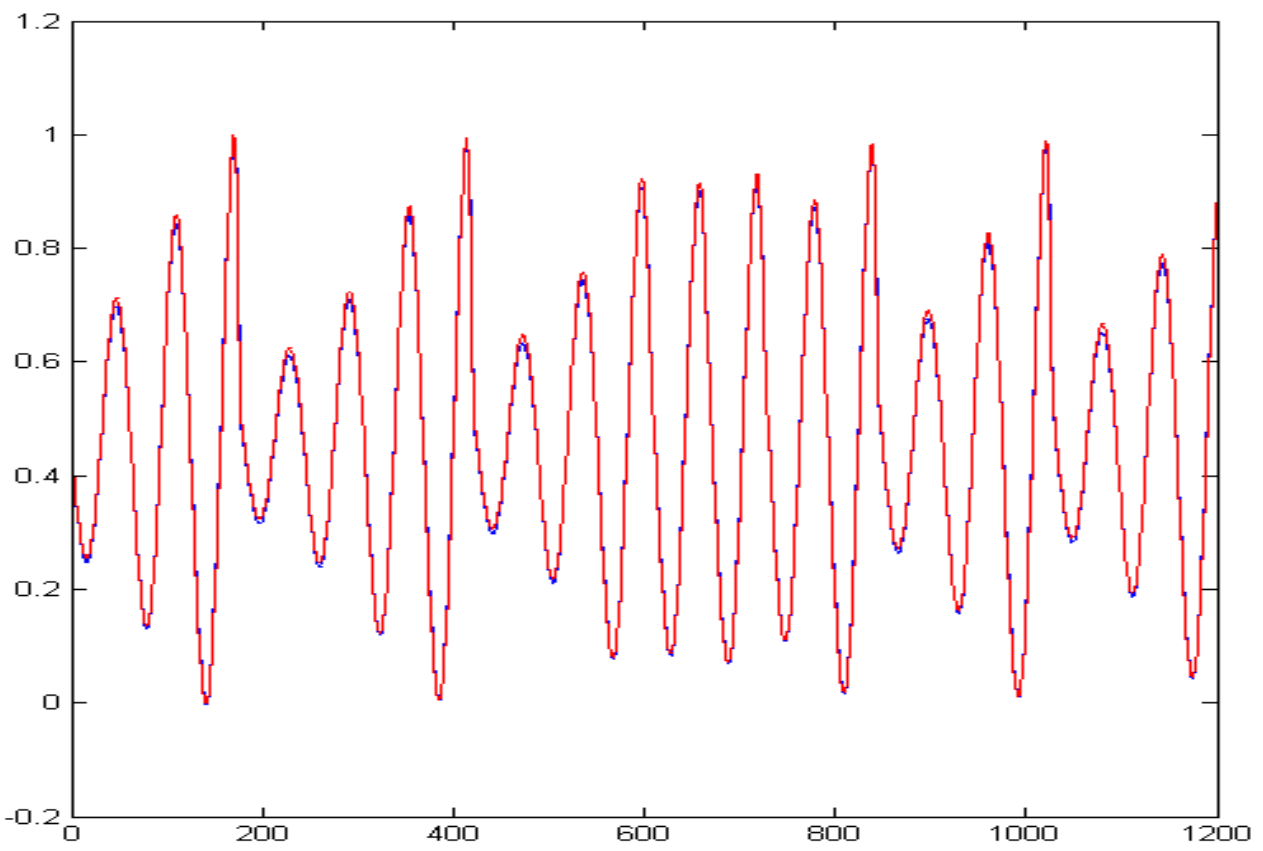

Figure 13. The predicted values and the target values of rossler time series (Train)

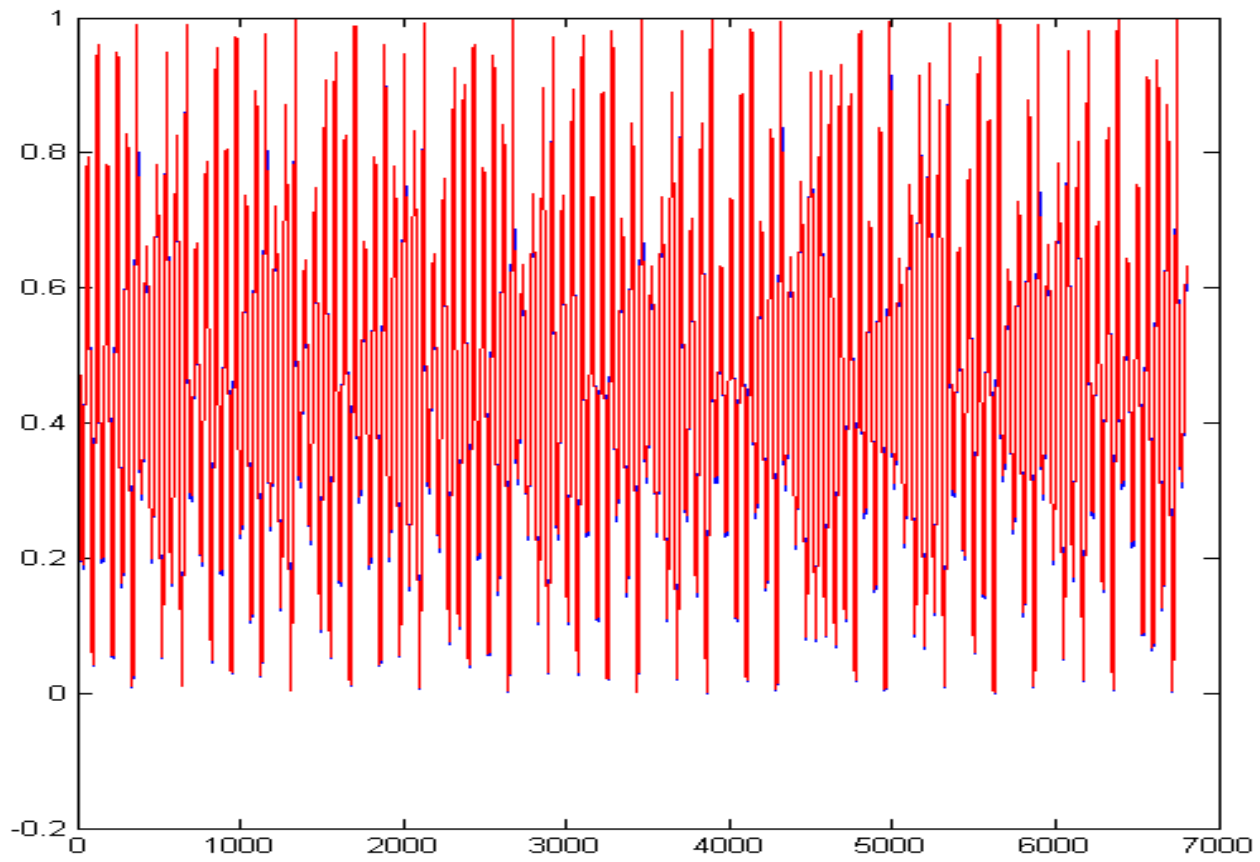

Figure 14. The predicted values and the target values of rossler time series (Test)

\section{Conclusion}

In this paper, an improved version of brain emotional learning algorithm based on the interval knowledge has been presented. The weights of the amygdala and orbitofrontal are updated based on interval knowledge. With regard to the implementation and performance of the improved brain emotional algorithm 
and its comparison with the original version to prediction the Lorenz and Rossler chaotic time series, a noticeable improvement in precision, accuracy and speed of convergence of the final results is reported.

\section{References}

[1] A. Ghezelbash, "Predicting Changes in Stock Index and Gold Prices to Neural Network Approach", The Journal of Mathematics and Computer Science, vol. 4 (2012), no. 2, pp. 227-236.

[2] M. Bagheri, M. Valipour, V. Amin, "The Bankruptcy Prediction in Tehran shareholding using Neural Network and it's Comparison with Logistic Regression," The Journal of Mathematics and Computer Science, vol. 5 (2012), no. 3, pp. 219-228.

[3] M. M. Javidi, M. H. Nattaj, "A New and Quick Method to Detect DoS Attacks by Neural Networks", The Journal of mathematics and computer Science, vol. 6 (2013), pp. 85-96.

[4] Z. Falahiazar, M. Rohani, A. Falahiazar, "Controlling the False Alarm in an Intrusion Tolerant Database System Using Significance Degrees of Data Objects", Journal of mathematics and computer science, vol. 13 (2014), pp. 212-225.

[5] Hagan, Martin T., Mohammad B. Menhaj. "Training feed forward networks with the Marquardt algorithm" Neural Networks, IEEE Transactions on 5, no. 6 (1994): 989-993.

[6] L. Falahiazar, M. Teshnehlab, A. Falahiazar, "Parallel Genetic Algorithm Based on a New", in International Conference on Recent Advances in Computing and Software Systems, (2012).

[7] Y. Sharafi, M. Ahmadieh Khanesar. Teshnehlab, "Discrete Binary Cat Swarm Optimization Algorithm", in Computer, Control \& Communication (IC4), $3^{\text {rd }}$ International Conference on, (2013).

[8] J. M. Jenkins, K. Oatley, N. L. Stein, "Human emotions: a reader", Blockwell, (1998).

[9] E.T. Rolls, "Neurophysiology and Functions of the Primate Amygdala. In: The Amygdala: Neurobiologycal Aspects of Emotion", Memory and Mental Dysfunction, (1992).

[10] J. Le Doux, "The Emotional Brain, Simon and Schuster", New York, (1996).

[11] J. L. McGaugh, F. Bermudez-Rattoni, R.A. Prado-Alcala, "Plasticity in the Central Nervous System: Learning and Memory", Lawrence Erlbaum Associates, Inc., Mahwah, NJ. (1995) 1739.

[12] J. Morén, C. Balkenius, "A Computational Model of Emotional Learning in the Amygdala, In: From Animals to Animats 6", Proceedings of the 6th International Conference on the Simulation of Adaptive Behaviour, Meyer, J.A., A. Berthoz, D. Floreano, H.L. Roitblat and S.W. Wilson (Eds.). MIT Press, Cambridge, MA. USA. (2000) 115-124.

[13] P. Lingras, "Rough Neural Networks", in: Proceedings of the Sixth International Conference on Information Processing and Management of Uncertainty, Granada, (1996) 1445-1450.

[14] S. Chandanal, V. Mayorga, "The New Rough Neuron", Neural Networks and Brain, Beijing, (2005) 13-18.

[15] C. Balkenius, J. Morén, "Emotional Learning: A Computational Model of Amygdala", Cybernetics and Systems, Vol 32; Part 6, (2001) 611-636.

[16] C. Lucas, D. Shahmirzadi, N. Sheikholeslami, "Introducing BELBIC: Brain emotional learning based intelligent controller", Int. J. Intell. Autom. Soft Comput. 10, (2004)11-21.

[17] E. Lotfi, M.R. Akbarzadeh, "Supervised Brain Emotional Learning”, Int. J. Intell. Autom. WCCI 2012 IEEE World Congress on Computational Intelligence, June, - Brisbane, Australia, (2012)1015. 